\title{
Philonsorbonne
}

1 | 2007

Année 2006-2007

\section{Questions concernant la morale de Bergson}

Iannis PRELORENTZOS

\section{(2) OpenEdition}

Journals

Édition électronique

URL : https://journals.openedition.org/philonsorbonne/129

DOI : 10.4000/philonsorbonne.129

ISSN : 2270-7336

\section{Éditeur}

Publications de la Sorbonne

\section{Édition imprimée}

Date de publication : 15 novembre 2007

Pagination : 103-129

ISBN : $978-2-85944-594-2$

ISSN : 1255-183X

\section{Référence électronique}

Iannis PRELORENTZOS, « Questions concernant la morale de Bergson », Philonsorbonne [En ligne], 1 । 2007, mis en ligne le 27 janvier 2013, consulté le 08 juin 2021. URL : http://journals.openedition.org/ philonsorbonne/129; DOI : https://doi.org/10.4000/philonsorbonne.129

(C) Tous droits réservés 


\section{Questions concernant la morale de Bergson}

\section{Iannis Prelorentzos}

Université de Ioannina (Grèce)

Cette étude porte sur la place de la morale dans l'œuvre de Bergson ; sur sa méthode en morale ; sur sa distinction fondamentale entre la morale close et la morale ouverte; enfin, sur le rôle du social et du biologique dans la morale bergsonienne .

\section{La place de la morale dans l'œuvre de Bergson}

Signalons d'emblée que Bergson ne distingue pas entre «morale » et « éthique » - il affirme même, après la publication des Deux Sources de la morale et de la religion (1932), qu'il n'avait jamais pensé à un tel contraste ${ }^{2}$.

Bergson s'est occupé tard de la morale (ainsi que de la religion et des questions sociales) dans sa carrière philosophique, puisque dans son premier ouvrage, l'Essai sur les données immédiates de la conscience (1889), il s'est surtout penché sur la psychologie, en examinant principalement les idées de «durée des états de conscience » et de liberté ; dans le second, Matière et mémoire (1896), sur les rapports entre la psychologie et la neurophysiologie (entre l'esprit et la matière ou entre l'âme et le corps) ; et dans le troisième,

1. Une première version de ce texte a été présentée en avril 2006 à L'École doctorale de philosophie de l'Université Paris I-Panthéon-Sorbonne dans le cadre du programme des échanges universitaires « Erasmus ». Je tiens à remercier Chantal Jaquet pour son invitation et pour l'organisation de cette rencontre.

2. Cf. Bergson, Correspondances, Paris, P.U.F., 2002, p. 1371 : «Le contraste dont vous parlez entre les résonances de notre mot "morale" et celles de l'anglais "ethics" - contraste auquel je n'avais jamais pensé - est devenu pour moi très frappant et très instructif depuis que vous me l'avez signalé ». 
L'Évolution créatrice (1907), sur la philosophie de la vie et sur la philosophie de la nature. Notons que, en 1910, il a jugé nécessaire de préciser à des correspondants qu'il ne pensait pas "avoir cédé, même inconsciemment, à des préoccupations morales en établissant [ses] pensées théoriques ${ }^{3}$ et qu'il a «philosophé en dehors de toute arrière-pensée religieuse $\gg{ }^{4}$.

Il est caractéristique que sa doctrine de la liberté dans l'Essai ne comporte pas de dimension morale; elle se situe au niveau métaphysique et la question des valeurs ne s'y pose pas ${ }^{5}$. Bergson va même jusqu'à reprocher "ironiquement à Kant son "scrupule moral", à cause duquel il avait "reconduit [la liberté] avec beaucoup d'égards dans le domaine des choses en soi" »". Ceci n'a évidemment pas échappé à ses contemporains (notamment à Lucien Lévy-Bruhl ${ }^{7}$ ); certains, notamment Gustave Belot, lui ont même reproché de ne pas avoir tiré les conséquences de sa conception de la liberté sur le plan moral ${ }^{8}$. Malgré ces critiques, Bergson procède de la même manière dans ses deux prochains grands livres ${ }^{9}$, puisque dans «Matière et mémoire et dans L'Évolution créatrice, il avait paru inscrire la liberté dans l'univers matériel et dans la création de la vie [...] sans lui assigner de direction ou de contenu moral ${ }^{10}$.

Ceci ne signifie pas qu'il n'y ait pas de thèmes ayant une connotation morale dans ses trois premiers grands ouvrages, comme par exemple celui de la création de soi par soi dans L'Évolution créatrice ${ }^{11}$, ou que Bergson n'ait pas eu une conception implicite de la morale comme réalisation de la volonté

3. Ibid., p. 329.

4. Ibid., p. 383.

5. Ph. Soulez et F. Worms, Bergson. Biographie, Paris, P.U.F., «Quadrige», 2002, p. 206207. Cf. aussi J.-L. Vieillard-Baron, Bergson, édition bilingue (grec-français), Athènes, Atrapos, 2001, p. 255 : «En réalité, la conception bergsonienne de la liberté [dans l'Essai] n'est pas immédiatement morale; elle est d'abord physique et métaphysique; [...] positivement [...] la liberté est l'expression d'un moi qui pose une action, indépendamment de savoir si cette action est bonne ou mauvaise. Le problème de la valeur n'est pas premier pour Bergson ».

6. Cf. Soulez et Worms, Bergson. Biographie, op. cit., p. 206-207.

7. Cf. une citation du compte rendu de l'Essai sur les données immédiates par L. Lévy-Bruhl in Soulez et Worms, Bergson. Biographie, op. cit., p. $76:$ : au lieu de prendre rang parmi les défenseurs ou les adversaires de la liberté morale, il a eu l'idée originale d'examiner l'énoncé même du problème et de voir s'il n'impliquait pas contradiction ».

8. Cf. ibid., p. 207 et notes 7 et 8 (p. 369-370).

9. Je précise : «grands livres », car Bergson lui-même qualifiait Le rire, publié en 1900 entre Matière et mémoire et L'Évolution créatrice, de « petit livre ».

10. Cf. Soulez et Worms, Bergson. Biographie, op. cit., p. 207.

11. Cf. L'Évolution créatrice, in Bergson, CEuvres, Édition du Centenaire, Paris, P.U.F., 1959, p. 500 : «On a donc raison de dire que ce que nous faisons dépend de ce que nous sommes; mais il faut ajouter que nous sommes, dans une certaine mesure, ce que nous faisons, et que nous nous créons continuellement nous-mêmes. Cette création de soi par soi est d'autant plus complète, d'ailleurs, qu'on raisonne mieux sur ce qu'on fait ». 
libre $^{12}$. Ceci ne signifie pas non plus que nous ne puissions pas reconstruire une doctrine bergsonienne de l'amitié dessinée durant les premières années après la première guerre mondiale, à travers des lettres personnelles et ses discours sur «l'amitié franco-américaine » ${ }^{13}$. Léon Brunschvicg a même essayé, en 1927, de relever «tous les jalons qui, depuis la doctrine de la liberté, font entrevoir chez Bergson une philosophie morale, du texte sur le "surhomme" de L'Évolution créatrice, à celui sur les "grands hommes de bien" de L'Énergie spirituelle $»^{14}$.

Cependant la morale est devenue un des objets principaux de la pensée de Bergson après la publication de L'Évolution créatrice (il l'affirme, par exemple, en $1909^{15}$ et en $1911^{16}$ et persiste dans cette affirmation en $1928^{17}$ ). Mais les problèmes moraux, qui sont inséparables des questions sociales et

12. Cf. J.-L. Vieillard-Baron, Bergson, édition bilingue (grec-français), op. cit., p. 256 : «Sans doute le thème de la création de soi par soi, c'est-à-dire de l'effort pour créer son propre caractère, a-t-il une connotation morale. On pourrait dire que Bergson a une conception implicite de la morale comme réalisation de la volonté libre ». Le même commentateur attribue une très grande importance au thème de la création de soi par soi dans l'orientation intellectuelle de Bergson après la publication de L'Évolution créatrice: «Bergson a mis très longtemps avant de livrer au public Les deux sources. On sait qu'après L'évolution créatrice, il songeait à un ouvrage dont il ne savait pas s'il porterait sur l'art ou sur la morale. La découverte progressive de l'importance de la mystique a fait pencher la balance vers la morale et la religion. La supériorité de la création de soi par soi, acte moral par excellence, sur la création d'une œuvre d'art, est affirmée en 1919, à la fin du texte sur «La conscience et la vie » (Euvres, p. 833) ; elle n'était qu'esquissée dans la conférence de 1911 (Mélanges, p. 932). La pensée de Bergson s'est donc affirmée en s'orientant conjointement vers la morale et la religion » (J.-L. Vieillard-Baron, Bergson, Paris, P.U.F., « Que sais-je ? », $2^{\mathrm{e}}$ édition 1993, p. 85-86). - Sur le thème bergsonien de la création de soi par soi, cf. l'article de Michel Jouhaud, «Bergson et la création de soi par soi », Les Études philosophiques, avriljuin 1992, p. 195-215.

13. F. Worms résume cette doctrine de l'amitié dans Soulez et Worms, Bergson. Biographie, op. cit., p. 202 : références fréquentes de Bergson à Aristote qui lui permettent « de distinguer divers degrés d'amitié selon l'objet de la relation même [...] Il ne s'agit pour Bergson ni de simple loisir partagé, ni pourtant non plus d'influence mutuelle : l'harmonie désigne un accord immédiat et sans distance entre des personnes qui restent pourtant des individus, métaphysiquement distincts. Pas plus qu'avec d'autres doctrines philosophiques, on ne saurait doser ici les interactions : l'individualité ou plutôt la personnalité reste un absolu ».

14. Ibid., p. 209. Brunschvicg fait cet effort dans le deuxième tome de son ouvrage Progrès de la conscience. " Il reproche pourtant à Bergson de ne pas donner à la conscience le moyen de tirer d'elle-même et de sa liberté la portée morale qui donnerait un sens à ses avancées théoriques et scientifiques » (ibid.).

15. Cf. ibid., p. 285 : «...c'est le problème religieux (avec ses harmoniques morales et sociales) qui se pose à moi maintenant, étant donné le point où mes réflexions m'ont conduit » (27 juillet 1909 ; c'est Bergson qui souligne).

16. Cf. Bergson, Mélanges, Paris, P.U.F., 1972, p. 881 : «La Morale ? Oui, je m’y intéresse. Évidemment, c'est là où je voudrais aboutir ». Cependant, dans le même entretien (avec Joseph Lotte) il semble hésiter entre l'esthétique et la morale : «L'Esthétique aussi me retient. Je travaille beaucoup. Esthétique, morale, il doit y avoir parenté, il doit y avoir des points communs » (ibid.).

17. Cf. Correspondances, op. cit., p. 1265 : «Ce sont les questions morales qui m'ont le plus occupé moi-même dans ces dernières années ». 
du problème religieux selon lui ${ }^{18}$, l'occupaient pendant des années sans qu'il puisse arriver à des conclusions satisfaisantes - et cela non seulement pour des raisons conjoncturelles; je me réfère à ses activités diplomatiques officieuses durant la première guerre mondiale, notamment à ses missions aux États-Unis ${ }^{19}$. Lui-même avait déclaré en 1912 qu'il n'était pas sûr de jamais rien publier au sujet de la morale ${ }^{20}$; en 1934 il nous confie qu'en 1922 il n'était «pas encore en possession complète des résultats » qu'il a exposés dans Les Deux Sources ${ }^{21}$; et en 1926 il n'était toujours pas sûr d'avoir le temps et la force d'achever un travail sur les questions morales ${ }^{22}$, en affirmant que la morale qu'il concevait restait encore imprécise ${ }^{23}$. Notons que certains de ses adversaires s'étaient empressés d'affirmer qu'il n'allait jamais pouvoir constituer une théorie morale (je pense notamment à un de ses adversaires les plus connus, Georges Politzer ${ }^{24}$ - dont la lecture de Bergson a fortement influencé la réception de la pensée bergsonienne par des philosophes comme Jean-Paul Sartre ou Merleau-Ponty ${ }^{25}$ - mais aussi à Jacques Maritain ou à Gustave Rodrigues ${ }^{26}$ ).

Une des raisons principales pour lesquelles Bergson s'est penché tard sur les questions morales et religieuses a été le fait que, bien qu'il considérât le problème de la durée ou de la personnalité comme le problème central de

18. Cf. ibid., p. 277: «Je n'ai rien écrit sur les questions sociales, non plus d'ailleurs que sur les problèmes moraux, qui en sont inséparables. C'est de ce côté-là que je me tourne en ce moment » (20 juillet 1909).

19. Cf. Mélanges, op. cit., p. 1554-1570. Sur les fameuses Missions de Bergson, cf. les analyses précieuses de $\mathrm{Ph}$. Soulez, Bergson politique, Paris, P.U.F., «Philosophie d'aujourd'hui », 1989, p. 89-125 (La première mission) et p. 175-207 (La deuxième mission).

20. Cf. Mélanges, op. cit., p. 964 : «Mais pour préciser encore plus ces conclusions [sur Dieu] et en dire davantage, il faudrait aborder des problèmes d'un tout autre genre, les problèmes moraux. Je ne suis pas sûr de jamais rien publier à ce sujet ».

21. Cf. sa note à la fin de la deuxième partie de l'Introduction à La pensée et le mouvant, in Euvres, p. 1330, note 1.

22. Cf. Correspondances, op. cit., p. 1178 : «je préférerai ne pas voir annoncer un grand travail de moi "sur les questions morales et sociales avec, peut-être, une ouverture sur la question religieuse" [...] Je ne suis pas sûr, malheureusement, d'avoir maintenant le temps et la force de venir à bout de ce travail. En tout cas, il ne faudrait pas laisser croire qu'il visera la question religieuse, car c'est de morale seulement qu'il s'agira ».

23. Cf. ibid., p. 1186 : «La morale que je conçois (d'ailleurs imprécise encore) [...] ».

24. Cf. Soulez et Worms, Bergson. Biographie, op. cit., p. 212.

25. Cf. Florence Caeymaex, Sartre, Merleau-Ponty, Bergson. Les phénoménologies existentialistes et leur héritage bergsonien, Hildesheim-Zürich-New York, Georg Olms Verlag, «Europaea Memoria », 2005, p. 7-9.

26. Cf. Blaise Romeyer, «Morale et religion chez Bergson», in Bouilhé, Bremond et al., Études d'histoire de la philosophie, Paris, Beauchesne, 1932, p. 283-317. L'auteur se réfère (p. 283-284) aux thèses développées, premièrement, par Gustave Rodrigues dans son étude Bergsonisme et Moralité (Paris, Chiron, 1922), suivant lequel Bergson ne pouvait pas édifier une morale s'il restait fidèle à sa méthode, puisque l'anti-moralisme constitue l'essence de l'œuvre bergsonienne ; et, deuxièmement, par Jacques Maritain, qui, dans La philosophie bergsonienne (Paris, Rivière, $2^{\mathrm{e}}$ édition 1930), soutenait que, contrairement au bergsonisme d'intention, le bergsonisme de fait était un nihilisme. 
la métaphysique ${ }^{27}$, il tenait les questions morales et religieuses pour «ce qu'il y a de plus difficile en philosophie $»^{28}$. Par ailleurs, sa conviction constante était que «la difficulté principale [du monde actuel] est morale, encore plus que politique ou économique, et qu' aucun résultat viable ne peut être obtenu sans une réforme morale de l'humanité $»^{29}$.

Une autre raison de poids que nous devons invoquer est le fait que Bergson ne concevait nullement la philosophie comme un système et que, par conséquent, il ne voulait aucunement déduire logiquement la morale de ce qu'il avait écrit auparavant sur d'autres problèmes : sur la durée, la liberté, la mémoire et surtout sur l'élan vital ${ }^{30}$. Par ailleurs, il a plus d'une fois déclaré qu'on n'est jamais tenu de faire un livre ${ }^{31}$, signifiant par là qu'il publierait un ouvrage sur la morale uniquement s'il considérait qu'il avait mené à bonne fin ses réflexions sur ce domaine ${ }^{32}$. Pour cette raison, lorsque Jacques Chevalier a consacré dans son ouvrage sur Bergson de 1926 un chapitre aux questions morales et religieuses, Bergson lui a demandé de préciser que ce qu'il y avançait n'engageait que lui, le commentateur ${ }^{33}$.

27. Cf. sa première leçon consacrée à l' «histoire de l'idée du temps » au Collège de France (année universitaire 1902-1903) in F. Worms (éd.), Annales bergsoniennes, Paris, P.U.F., «Épiméthée », vol. I : «Bergson dans le siècle », 2002, p. 45 : «le problème de la durée est le problème central de la métaphysique ». Cf. également la première conférence de Bergson sur la personnalité à l'Université d'Edinburgh, in Mélanges, op. cit., p. 1051-1052 et p. 1071 : «On peut considérer le problème de la personnalité comme le problème central de la philosophie ».

28. Correspondances, op. cit., p. 1386 : «Ces questions morales et religieuses qui sont, de beaucoup, ce qu'il y a de plus difficile en philosophie». Cf. aussi ibid., p. 1233 (23 sept. 1927): "Ces questions morales sont les plus difficiles de toutes, et elles ont été singulièrement négligées par la philosophie moderne ».

29. Ibid., p. 1393-1394 : en 1932, il affirme au colonel House qu'il continue d'avoir cette conviction qui était la sienne durant la première guerre mondiale.

30. Cf. par exemple ibid., p. 286 : «La philosophie, telle que je la conçois, ne constitue pas un système; elle ne permet pas de résoudre une question nouvelle au moyen des solutions qu'on a déjà données des autres problèmes » (c'est Bergson qui souligne) ; cf. également ibid., p. 277. Cf. aussi la lettre de Bergson à Édouard Le Roy, du 30 oct. 1912, publiée par J.-L. Vieillard-Baron dans son article «Lettres inédites de Bergson», Annales bergsoniennes, vol. II : « Bergson, Deleuze, la phénoménologie », Paris, P.U.F., coll. «Épiméthée », 2004, p. 473-474. - En quoi les thèses essentielles des Deux Sources ne sont pas une déduction pure et simple de ce que Bergson avait écrit auparavant, notamment de sa philosophie de la vie développée dans L'Évolution créatrice, mais comportent des solutions inattendues, imprévisibles? Cf. F. Worms, Bergson ou les deux sens de la vie, Paris, P.U.F., «Quadrige/Essais. Débats », 2004, p. 269-272 et 316-317.

31. Cf. par exemple la dernière phrase de l'introduction bipartite de La Pensée et le mouvant, in Euvres, p. 1330.

32. En effet, Bergson n'a jamais publié un ouvrage sans avoir été « en possession complète de [ses] résultats » : cf. la note à la fin de la deuxième partie de l'introduction de Bergson à $L a$ pensée et le mouvant, in Euvres, p. 1330, note 1.

33. Cf. Soulez et Worms, Bergson. Biographie, op. cit., p. 206. Signalons que «Albert Thibaudet avait consacré aux problèmes moraux et religieux certains chapitres exploratoires [...] de son Bergsonisme. Nul n'était plus conscient, cependant, de leur caractère provisoire » (ibid.). 
Enfin, Bergson considérait que «les idées réellement viables, en philosophie, sont celles qui ont été vécues d'abord par leur auteur - vécues, c'est-à-dire appliquées par lui, tous les jours, à un travail qu'il aime, et modelées par lui, à la longue, sur cette technique particulière ${ }^{34}$.

\section{Pourquoi était-il selon Bergson si important, sinon essentiel, d'écrire un ouvrage sur la morale ?}

À part son intérêt personnel pour ce genre de questions et à part l'intérêt croissant qu'excitaient les problèmes moraux au début du vingtième siècle ${ }^{35}$, il a dû sentir le besoin de répondre à une série d'objections qu'on lui adressait. Je ne me réfère pas ici seulement aux réactions mentionnées provoquées par sa doctrine de la liberté dans l'Essai, mais surtout au fait que, «à mesure qu'on approche des années 1930, [les objections et les critiques adressées à Bergson] passent le plus souvent du terrain de la théorie de la connaissance, ou même de la philosophie morale ou politique, vers celui de la responsabilité morale et politique de la philosophie $»^{36}$.

À part «la critique adressée à Bergson par les auteurs (proches notamment de la tradition kantienne [comme Jean Nabert et Léon Brunschvicg]) qui demandent à la philosophie une règle rationnelle capable de guider une conscience morale, non seulement individuelle mais collective, non seulement dans une vie mais dans l'histoire ${ }^{37}$, il faut tenir compte des polémiques engagées avec Bergson : avec La trahison des clercs (1927), la polémique de Benda, vieille de quinze ans, «prend [...] un tour moral et politique $»^{38}$. Georges Politzer, de son côté, «revient à l'absence d'une doctrine morale» chez Bergson, comme nous l'avons vu: selon Politzer, «toute la vie [de Bergson], comme les indications qu'il a données de sa morale [...] nous permettent de comprendre qu'il s'est donné intégralement aux valeurs bourgeoises $»^{39}$.

34. Oeuvres, 1470. C'est F. Worms qui a attiré notre attention sur ce « texte trop méconnu, et essentiel » (Soulez et Worms, Bergson. Biographie, op. cit., p. 214).

35. Cf. Correspondances, op. cit., p. 347 : en 1910 Bergson attribue, du moins en partie, «l'intensité du mouvement philosophique » en France «depuis un certain nombre d'années » à «l'intérêt croissant qu'excitent les problèmes sociaux, moraux et religieux ».

36. Soulez et Worms, Bergson. Biographie, op. cit., p. 209.

37. Ibid.

38. Ibid., p. 210.

39. Ibid., p. 212 (c'est nous qui soulignons). 


\section{La morale définitive ${ }^{40}$ de Bergson : Les Deux Sources de la morale et de la religion (1932)}

Vingt-cinq ans après la publication de L'Évolution créatrice, à laquelle il devait sa renommée mondiale, et alors qu'il souffrait depuis sept années (depuis la fin de l'année 1924) d'un « violent rhumatisme articulaire ${ }^{41}$ qui ne lui permettait de travailler que très peu (difficilement et péniblement : au plus une heure par jour $)^{42}$, Bergson a enfin publié son ouvrage, tant attendu deux décennies plus tôt, sur la morale et la religion ${ }^{43}$. Ce fut une heureuse surprise pour beaucoup de gens - étant donné que Bergson n'avait «montré [son] manuscrit et [ses] épreuves à personne ${ }^{44}$ avant de les corriger ${ }^{45}-$ et l'événement a été salué comme une "victoire extraordinaire que l'esprit qui est en vous a obtenue sur le mal et toutes les puissances de douleur ${ }^{46}$.

\section{La méthode de Bergson dans Les Deux Sources}

Je vais me borner à cinq remarques sur la méthode suivie par Bergson dans cet ouvrage :

40. Bergson lui-même nous incite à parler de «morale définitive » dans son œuvre - par contraste, évidemment, avec la «morale par provision» de Descartes - lorsque, en 1901, il explique pourquoi il n'avait pas encore édifié «la morale précise et définitive » que lui demandait son contradicteur (Mélanges, op. cit., p. 487).

41. Cf. la lettre de Bergson à H. A. Lorentz, du 28 décembre 1924, in Correspondances, op. cit., p. 1136 : «Je suis immobilisé dans une position semi horizontale par une arthrite fort douloureuse. On pensait que ce ne serait rien ; mais les médecins refusent maintenant de faire un pronostic sur la date approximative où je pourrai sortir, ou tout au moins marcher». Cf. aussi ibid., p. 1139 (1 $1^{\text {er }}$ janvier 1925) et p. 1339 (25 janvier 1931) : «Il s'agit d'une affection rhumatismale d'une intensité rare, qui atteint toutes les articulations ».

42. Cf. notamment sa lettre à Floris Delattre, son neveu par alliance, du 11 mars 1929: «à compter maintenant par minutes, plutôt que par heures, le temps dont je dispose pour mon travail »(Mélanges, op.cit., p. 1493 et le même texte dans Correspondances, op. cit., p. 1295). Cf. également ibid., p. 1278, 1289, 1291, 1308, 1311, 1322, 1331, 1332, 1333, 1345, 1354,1380 et 1385 .

43. Sur le fait que plusieurs amis et disciples de Bergson (Édouard Le Roy, Charles Péguy, Albert Thibaudet, Jacques Chevalier) attendaient un livre de lui sur la morale et la religion après la publication de L'Évolution créatrice, cf. Blaise Romeyer, «Morale et religion chez Bergson », op. cit., p. 284. Joseph Lotte croyait même que la publication du «livre de Morale » de Bergson était imminente en 1911 ! (cf. Mélanges, op. cit., p. 880).

44. Ce qu'il regrette, écrit-il à J. Chevalier, car il aurait «ainsi évité [...] une ou deux expressions équivoques ». Cependant, au fond, il ne regrette pas d'avoir agi ainsi, car, «si je m'étais mis à discuter avec moi-même sur mon manuscrit une fois arrêté, je ne l'aurais peutêtre pas publié tant j'en avais peu envie » (Correspondances, op. cit., p. 1365). Cf. aussi ibid., p. 1363 : «Il aurait suffi de bien peu de chose, d'une observation légère qui eût à peine été une critique, pour me faire renoncer à la publication. C'est une des raisons pour lesquelles je n'ai rien montré à personne, ni des épreuves, ni du manuscrit ».

45. Cf. ibid. (à J. Chevalier, 25 février 1932).

46. Lettre de Paul Valéry à Bergson du 8 mars 1932 in Bergson, Mélanges, op. cit., p. 1499. 
a/ Bergson soutient à plusieurs reprises que sa contribution essentielle à la philosophie, c'est sa méthode ${ }^{47}$. Il affirme, plus précisément, que, s'il a «apporté quelque chose de nouveau en philosophie, c'est l'idée qu'on ne peut passer de la solution d'un problème à celle d'un autre par voie d'extension et de généralisation, et qu'il faut oublier, devant la nouvelle question qui se pose, tout ce qu'on a pu penser sur d'autres sujets ${ }^{48}$. Bergson considère comme l'apport majeur des Deux Sources «la proposition d'une nouvelle méthode, l'idée de poser autrement le problème ${ }^{49}$.

\section{b/ Bergson répète à satiété :}

Positivement, que, en tant qu'il fait œuvre de philosophe (et non de théologien $)^{50}$, il s'appuie uniquement sur l'expérience ${ }^{51}$ - parfois il ajoute : «sur l'expérience aidée du raisonnement $»^{52}$, et quelquefois il précise : du «raisonnement fondé sur l'expérience $»^{53}$. Mais il faut souligner ici que Bergson emploie «expérience » au sens large du terme, qui comprend non seulement les données des sens externes, mais aussi tant l'expérience intérieure, c'est-à-dire l'introspection ${ }^{54}$ - qui peut être également pratiquée

47. Cf. à titre d'exemple Correspondances, op. cit., p. 1187.

48. Ibid., p. 1075.

49. Cf. le texte des deux feuillets glissés par Bergson dans son exemplaire de l'ouvrage critique de Loisy, Y a-t-il deux sources de la morale et de la religion ? (1933, $2^{\mathrm{e}}$ éd. 1934). Ce texte fut publié dans les Annales bergsoniennes, vol. I, op. cit., p. 135.

50. Cf. Correspondances, op. cit., p. 1184 : «[...] avec la méthode de la philosophie pure telle que je l'entends (je ne parle pas, bien entendu, de l'appel à la révélation et de la foi) $[\ldots] »$.

51. Cf. à titre d'exemple les passages suivants des Deux Sources: «nous nous en tenons à l'expérience» (p. 83); «l'expérience ne dit rien de semblable» (p. 108); «nous avons essayé d'établir [...] en serrant d'aussi près que possible le contour des faits» (p. 335). Cf. aussi les passages suivants puisés dans les Correspondances de Bergson : «Je ne vais pas aussi loin. C'est dépasser le donné de l'expérience pure (à laquelle je prétends m'en tenir) » (p. 1182); «j'évite les explications par la cause première, qui sont nécessairement des constructions, alors que je prétends m'en tenir à l'expérience [...] Cette interprétation détruirait ce que je tiens pour essentiel dans la doctrine, et surtout dans la méthode. Celle-ci consiste à partir de l'expérience, et à remonter aussi haut que possible vers la source, mais à s'arrêter là où s'arrête l'expérience » (p. 1183). Nos références aux Deux Sources renvoient à la pagination de la $3^{\mathrm{e}}$ édition «Quadrige » de l'ouvrage, en 1988.

52. Cf. ibid., p. 1365 : «Mon livre est en effet un livre de philosophie. Il est entendu que, pendant que je l'écris, je n'admets d'autre source de vérité que l'expérience et le raisonnement $»$.

53. Cf. ibid., p. 1380: «la philosophie [...] sa méthode à elle étant expérience, et raisonnement fondé sur l'expérience». Selon Bergson le raisonnement, c'est-à-dire l'induction et la déduction, est la fonction essentielle de l'intelligence (cf. ibid., p. 315). En outre, en 1905, Bergson soutenait, en renvoyant à son essai «Introduction à la métaphysique », que le fait qu'une de ses distinctions repose sur des raisonnements n'entraîne pas «que ce ne soit pas une donnée de l'intuition », le rôle de la raison étant « de préparer et donc de confirmer l'intuition par la dialectique » (ibid., p. 114 ; c'est l'auteur qui souligne).

54. Cf. par exemple les passages suivants des Deux Sources: «N'oublions pas que nous cherchons au fond de l'âme, par voie d'introspection, les éléments constitutifs d'une religion 
en quelque sorte sur autrui, «le psychologue se transportant par sympathie à l'intérieur d'une conscience qui n'est plus la sienne $»^{55}$ - que l'expérience (du contact, de l'union avec le divin) des grands mystiques ${ }^{56}$.

Il faut également ajouter ici que, lorsqu'il nous invite à nous en tenir à l'expérience, il ajoute parfois : «et aux probabilités qu'elle suggère ${ }^{57}$.

Deux remarques s'imposent ici :

- Le fait que Bergson s'appuie sur l'introspection, qui peut être également pratiquée en quelque sorte sur autrui à travers le procédé de la sympathie, est très important en morale, car Bergson tire deux conséquences majeures du rejet de la théorie de l'hérédité des habitudes acquises ${ }^{38}$, rejet

primitive » (p. 137-138); « un examen attentif de ce qui se passe dans notre conscience nous montre que [...]» (p. 130); «suivons donc notre méthode habituelle. Demandons à notre propre conscience, débarrassée de l'acquis, rendue à sa simplicité originelle [...] L'observation de soi est ici fort difficile [...]»(p. 160). - Cf. également ce que Bergson écrivait à Édouard Le Roy le 30 oct. 1912: «La difficulté que certains de mes critiques trouvent à concilier ensemble telles ou telles de mes vues vient surtout, je crois, de ce qu'ils veulent les rattacher à un principe d'où elles auraient été déduites systématiquement. J'ai procédé d'une manière toute différente, en suivant pas à pas l'expérience - l'expérience intérieure d'abord, mais l'expérience du dehors également. Je n'ai pas eu d'autre principe, et là est tout mon "système" " (cette lettre, qui ne fait pas partie des Correspondances de Bergson, fut publiée par J.-L. Vieillard-Baron dans son article «Lettres inédites de Bergson », Annales bergsoniennes, vol. II, op. cit., p. 473-474).

55. Cf. l'éloge de William James par Bergson dans Mélanges, op. cit., p. 1544 : «Mais là n'est pas le principal intérêt du livre [de W. James], et tout autre en est la méthode fondamentale. Cette méthode est tout simplement la vieille méthode d'introspection, longtemps jugée imprécise et stérile, et que James reprit pour en tirer des résultats qu'on n'avait jamais osé espérer. C'était l'introspection pratiquée sur soi-même et aussi, en quelque sorte, sur autrui, le psychologue se transportant par sympathie à l'intérieur d'une conscience qui n'est plus la sienne, et coïncidant avec elle temporairement. Envisagée de ce côté, comme mettant en œuvre l'observation de soi par soi, et l'observation d'autrui par l'élargissement de soi, la méthode psychologique fit que William James fut l'égal des plus grands philosophes de tous les temps ». Cf. aussi ibid., p. 1471. - Sur le sens bergsonien du terme «sympathie» dans ce cadre, cf. ibid., p. 1550 et Correspondances, op. cit., p. 1347 : l'étymologie exige que la sympathie soit «coïncidence partielle avec autrui, une pénétration». - Ailleurs aussi Bergson lie l'introspection à la possibilité de donner des coups de sonde dans l'âme d'autrui. En effet, il félicite Charles Du Bos de ses Extraits du Journal (1908-1928) en disant : «En un sens, c'est bien de l'introspection (pour employer un mot que nous avons emprunté à la psychologie anglaise). Mais c'est aussi bien ce que les Anglais appelleraient de l' «insight», car vous donnez des coups de sonde dans l'âme d'autrui en même temps que dans la vôtre » (Correspondances, op. cit., p. 1295).

56. Sur ce point, Bergson doit sans doute beaucoup à la façon dont William James n'a rien sacrifié de l'expérience dans son ouvrage Les Variétés de l'expérience religieuse. Cf. ce que Bergson en dit dans Mélanges, op. cit., p. 1472.

57. Cf. Deux Sources, p. 290 : «Tenons-nous en donc aux faits que l'on constate et aux probabilités qu'ils suggèrent ».

58. Sur le rejet de l'hérédité de l'acquis dans Les Deux Sources, cf. p. 83, 117, 132, 134, 167 168, 181, 290. Cf. un texte très caractéristique : «nous nous en tenons à l'expérience, qui nous montre dans la transmission héréditaire de l'habitude contractée une exception - à supposer qu'elle se produise jamais - et non pas un fait assez régulier, assez fréquent, pour déterminer à la longue un changement profond de la disposition naturelle » (ibid., p. 83). 
qu'il considère comme «un des deux ou trois points essentiels des Deux Sources: (a) L'homme primitif subsiste en nous et nous pouvons le retrouver; (b) il n'y a rien à faire en morale, en politique etc., si l'on ne va pas le chercher, le décrire, l'interroger, pour découvrir le moyen de tourner ses exigences une à une, ou (ce qui vaudrait infiniment mieux) le neutraliser tout d'un coup » ${ }^{59}$.

- Bergson s'empresse de souligner qu'il tient pour la contribution principale des Deux Sources le fait qu'il a montré aux philosophes «qu'il existe une certaine expérience, dite mystique, à laquelle ils doivent, en tant que philosophes, faire appel ou dont ils doivent tout au moins tenir compte. Si j'apporte, dans ces pages, quelque chose de nouveau, c'est cela : je tente d'introduire la mystique en philosophie comme procédé de recherche philosophique ${ }^{60}$.

Dans une lettre adressée à Henri Gouhier, Bergson met en valeur le rôle fondamental de l'expérience dans sa philosophie, en montrant comment il est passé de L'Évolution créatrice aux Deux Sources : «Il ne faut pas perdre de vue que mon objet a toujours été d'introduire l'expérience en métaphysique. Quand je parle d'un "élan vital", je tâche de serrer d'aussi près que possible les données empiriques de la biologie. Quand j'aborde l'étude de Dieu, je me réfère à l'expérience mystique. Nous tenons solidement ainsi "les deux bouts de la chaîne", et nous ne sommes tout à fait sûr que lorsque nous spéculons sur ces deux points sans trop nous écarter d'eux $»^{61}$.

Cependant, étant donné le rôle fondamental de l'expérience dans la philosophie de Bergson - rappelons, premièrement, que, dans Matière et mémoire, il nous invite «à aller chercher l'expérience à sa source, ou plutôt au-dessus de ce tournant décisif où, s'infléchissant dans le sens de notre utilité, elle devient proprement l'expérience humaine $»^{62}$, deuxièmement, qu'il termine l'Introduction à la métaphysique en définissant la métaphysique comme «expérience intégrale ${ }^{63} \mathrm{et}$, troisièmement, qu'il voit «l'avenir de la psychologie, et même de la philosophie, dans un retour à l'expérience directe ${ }^{64}$ - et, durant à peu près la même période, dans le pragmatisme de William James et dans la phénoménologie allemande

59. Cf. dans les Annales bergsoniennes, vol. I, op. cit., p. 134 le texte des deux feuillets glissés par Bergson dans son exemplaire de l'ouvrage critique de Loisy, $Y$ a-t-il deux sources de la morale et de la religion? Sur la manière dont nous pouvons retrouver l'humanité primitive en nous, cf. Deux Sources, p. 132-133, 140 et 167-168.

60. Correspondances, op. cit., p. 1365 (c'est Bergson qui souligne). Immédiatement après, il se hâte de dissocier son emploi du mysticisme de la foi à un dogme : «Je suis donc tenu de démontrer qu'il n'y a pas solidarité entre l'acceptation de cette méthode de recherche et la foi à un dogme, quel qu'il soit. Et le seul moyen de le montrer est de supposer un instant le dogme aboli, et de constater que la méthode conserve toute sa valeur, toute sa force ».

61. Correspondances, op. cit., p. 1377.

62. Cuvres, p. 321.

63. Ibid., p. 1432.

64. Correspondances, op. cit., p. 1188-1189 (c'est nous qui soulignons). 
(Husserl) et bientôt française (Sartre, Merleau-Ponty), il faut souligner que suivre Bergson lorsqu'il nous invite à nous fier à l'expérience constitue plutôt un problème à résoudre qu'une solution. Je me réfère notamment à la question que pose Jean-Paul Sartre à propos de l'expérience en psychologie $^{65}$, aux problèmes que pose le recours de Bergson au recoupement des « lignes de faits» dans son essai «La conscience et la vie $»^{66}$ et aux points discutés par Renaud Barbaras dans ses articles sur l'expérience bergsonienne : «Le tournant de l'expérience : Merleau-Ponty et Bergson ${ }^{67}$ et $«$ Le problème de l'expérience $»{ }^{68}$.

Négativement: Bergson s'oppose radicalement à toute construction théorique ${ }^{69}$, surtout en matière de morale ${ }^{70}$ même à la construction d'une morale avec des éléments empruntés à ses propres travaux ${ }^{71}(\ll[\ldots]$ la méthode que je propose excluant toute construction et arrêtant la recherche au point précis où s'interrompt l'expérience » $)^{72},-$ bien qu'il emploie, au moins une fois, le terme «construction » positivement, soulignant toutefois que dans cette construction les concepts doivent être remplacés par des intuitions $^{73}-$ et à toute abstraction ${ }^{74}$. Contre les morales théoriques, qui ne

65. Cf. Esquisse d'une théorie des émotions, Paris, Hermann, 1938, p. 7-8 : «La psychologie est une discipline qui prétend être positive, c'est-à-dire qu'elle veut tirer ses ressources de l'expérience exclusivement [...] Encore faut-il limiter ce concept d'expérience, lorsqu'on parle de la psychologie contemporaine, car il peut y avoir, en somme, une foule d'expériences diverses et, par exemple, on peut avoir à décider s'il existe ou non une expérience des essences ou des valeurs ou une expérience religieuse ».

66. Cf. F. Worms, Bergson ou les deux sens de la vie, op. cit., p. 262 : «s'agit-il vraiment d'un recoupement empirique, ou ne court-on pas le risque d'une analogie dogmatique, voire d'une image devenue métaphorique ?»

67. Cf. Philosophie, 54, 1997, p. 33-59 ; article repris dans R. Barbaras, Le tournant de l'expérience. Recherches sur la philosophie de Merleau-Ponty, Paris, Vrin, «Histoire de la philosophie », 1998, p. 33-61.

68. Cf. Annales bergsoniennes vol. II, op. cit., p. 287-303.

69. Il ne cesse de s'opposer à tous ceux qui conçoivent le travail philosophique comme une construction. Cf. par exemple Mélanges, op. cit., p. 1544.

70. Cf. ibid., p. 881 : «Les idées générales, les morales théoriques ne servent à rien. On a fait de très belles constructions dans ce genre. En pratique, c'est nul ».

71. En 1926 il écrit à J. Chevalier : «Il y a ici une morale religieuse construite avec des éléments empruntés à mes travaux ; mais je ne l'ai pas construite moi-même, comme le lecteur pourrait le croire. La morale que je conçois (d'ailleurs imprécise encore) ne va pas contre cet esprit, mais serait plus complexe » (Correspondances, op. cit., p. 1186).

72. Mélanges, op. cit., 1504. Cf. aussi Correspondances, op. cit., p. 1183 : «J'évite les explications par la cause première, qui sont nécessairement des constructions, alors que je prétends m'en tenir à l'expérience [...] Cette interprétation détruirait ce que je tiens pour essentiel dans la doctrine, et surtout dans la méthode. Celle-ci consiste à partir de l'expérience, et à remonter aussi haut que possible vers la source, mais à s'arrêter là où s'arrête l'expérience ». Cf. également ibid., p. $1185:$ « [...] ma méthode, qui est de ne jamais recourir à la Cause première et de toujours donner des explications spécifiques ». Cf. aussi ibid., p. 1187.

73. Cf. ibid., p. 1378. 
servent à rien, Bergson voulait édifier une morale «qui serve à la pratique $\gg$. Au niveau de la morale, son adversaire constructiviste principal est Kant. Il est piquant de constater qu'une des critiques principales adressées par G. Politzer à Bergson était que sa psychologie et sa métaphysique restaient abstraites par rapport à la «psychologie concrète » du même Politzer ${ }^{76}$.

Selon Bergson, penser sur la morale ne suffit pas ; pour écrire quelque chose de solide dans ce domaine, il faut absolument l'avoir senti et vécu en même temps que pensé ${ }^{77}$.

En outre, il s'oppose bien évidemment aux préjugés, aux illusions, aux idées toutes faites ${ }^{88}$ et à toute idée préconçue ${ }^{79}$, en soulignant que les questions morales sont tout particulièrement « un sujet qu'on ne traite guère sans idées préconçues et convenues ${ }^{80}$.

c/ Suivant un des procédés méthodologiques essentiels des Deux Sources, qui rappelle par certains côtés la fameuse dernière proposition du Tractatus logico-philosophicus de Wittgenstein ${ }^{81}$, il faut garder le silence partout où l'expérience aidée du raisonnement ne nous permet pas de nous prononcer avec certitude sur tel ou tel sujet. La discussion aurait-elle pu l'aider à atteindre la certitude ? Non, car « elle force l'auteur à préciser trop tôt sa pensée sur bien des points, à la préciser par un effort purement dialectique et artificiel, alors que c'est par expérience graduelle et par maturation naturelle que des idées devraient se préciser ${ }^{82}$. Bergson admet à

74. Cf. ibid., p. 1095-1096 : Bergson s'oppose aux « habitudes de pensée abstraite que vous avez puisées dans la philosophie » et loue la «vision concrète des choses, dans leur détail changeant et mouvant». Cf. également ibid., p. 242 et 266.

75. Cf. Mélanges, op. cit., p. 881.

76. Cf. Soulez et Worms, Bergson. Biographie, op. cit., p. 211-212.

77. Cf. Correspondances, op. cit., p. 1265.

78. Cf. Mélanges, op. cit., 1553 : «percer la croûte épaisse des illusions, des idées toutes faites [...]». Cf. aussi Correspondances, op. cit., p. 1348 : il félicite J. E. Blanche de la «résolution prise de ne jamais regarder à travers des idées toutes faites, mais de voir les choses directement ». Cf. aussi ibid., p. 1350.

79. Cf. Deux Sources, p. 335 : « Nous avons essayé d'établir, en écartant les idées préconçues qu'on accepte des deux côtés [...] »Cf. aussi Mélanges, op. cit., p. 1472 et Correspondances, op. cit., p. 1301: «Philosophie et science se sont rencontrées ici parce qu'elles étaient décidées, l'une et l'autre, à écarter les idées préconçues et à se replacer en présence des faits ».

80. Correspondances, op. cit., p. 1265.

81. La confrontation autrement plus importante de l'analyse bergsonienne des conditions de possibilité d'un discours signifiant avec celle du Tractatus de Wittgenstein a été menée par J.-C. Pariente dans son étude magistrale «Bergson et Wittgenstein » (Revue internationale de philosophie, $\mathrm{n}^{\circ} 88-89,1969$, p. 183-204).

82. Ibid., p. 282. Il serait intéressant de comparer l'opposition de Bergson à « la controverse publique en matière de philosophie pure » (cf. ibid.) avec l'attitude négative de Gilles Deleuze envers la discussion en philosophie (cf. à titre d'exemple Pourparlers 1972-1990, Paris, Les Éditions de Minuit, 1990/2003, p. 191 : «la philosophie n'a strictement rien à voir avec une discussion, on a déjà assez de peine à comprendre quel problème pose quelqu'un et comment 
plusieurs reprises qu'il a préféré laisser des lacunes au lieu de juxtaposer «des opinions simplement personnelles et conjecturales » à des conclusions dont il se sentait certain ${ }^{83}$. Pour cette raison, la «révélation qui a une date » n'a pas de place dans Les Deux Sources, sans pourtant en être exclue. Seulement Bergson ne peut pas en tenir compte en tant que philosophe ${ }^{84}$.

Bergson admet un seul moyen pour «combler ces lacunes»: non pas par des concepts mais par des intuitions :

Quand je n'en dis pas davantage, c'est que je n'en sais pas davantage, le mot "savoir" étant entendu au sens que j'ai voulu lui donner en métaphysique. Mais, encore une fois, rien n'empêche de combler les lacunes par une construction, je veux dire par des explications dont les termes n'auront pas un sens plein et ne pourront se remplir que si les concepts y sont remplacés par des intuitions ${ }^{85}$.

d/ Nous savons que, afin de mener à bonne fin la composition d'un ouvrage, Bergson se documentait très sérieusement ${ }^{86}$. Pour cette raison notamment cela lui a pris six à sept ans pour écrire Matière et mémoire, presque onze ans pour composer L'Évolution créatrice et pas moins de vingt-cinq ans pour la rédaction des Deux Sources (selon ses propres dires) ${ }^{87}$.

Sur quelles données a-t-il réfléchi afin d'achever Les Deux Sources et quels ouvrages a-t-il lu ? Nous savons qu'il avait étudié, à part les grandes théories morales classiques (platonicienne, aristotélicienne, épicurienne, stoïcienne, kantienne, utilitariste etc.), divers ouvrages de ses contemporains consacrés à la morale (tels que les livres de Lucien Lévy-Bruhl et de

il le pose, il faut seulement l'enrichir, en varier les conditions, ajouter, raccorder, jamais discuter $\gg$ ).

83. Cf. Correspondances, op. cit., p. 1388-1389 : «Vous avez d'ailleurs bien raison de donner à entendre qu'il y a dans ce livre [dans Les Deux Sources] des lacunes. Elles tiennent, le plus souvent, à ce que je me tais quand je ne vois plus clair, ne voulant pas mêler à des conclusions dont je me sens certain des opinions simplement personnelles et conjecturales ». Cf. également Mélanges, op. cit., p. 1504: «il m'en faudrait beaucoup [de travail] pour répondre d'une manière satisfaisante aux questions qui ont surgi au cours de l'entretien [sur Les Deux Sources]. Telle d'entre elles, d'ailleurs, ne comporterait peut-être pas une solution complète, la méthode que je propose excluant toute construction et arrêtant la recherche au point précis où s'interrompt l'expérience » (c'est nous qui soulignons).

84. Cf. Correspondances, p. 1380 : «je n'ai nullement exclu "la révélation qui a une date". Je me suis borné à dire que la philosophie n'avait pas le droit de l'invoquer, sa méthode à elle étant expérience, et raisonnement fondé sur l'expérience ».

85. Ibid., p. 1378 (c'est nous qui soulignons).

86. Sur le rôle des informations et des réflexions dans la composition des livres de Bergson, cf. Mélanges, op. cit., p. 881 et Correspondances, op. cit., p. 314, 409 et 444.

87. Cf. ibid., p. 1428-1429: en réagissant à la critique exercée par A. Loisy aux Deux Sources, Bergson se réfère à ce livre comme à «un ouvrage philosophique écrit après 25 ans de recherches et de réflexion ». 
Frédéric Rauh ${ }^{88}$, de Benedetto Croce ${ }^{89}$, de son collègue Alfred Loisy ${ }^{90}$ ) ou portant en partie sur la morale ${ }^{91}$, qu'il avait apprécié le travail de John Dewey sur l'éthique et qu'il s'intéressait «au mouvement de culture éthique »du professeur américain Felix Adler ${ }^{92}$.

Il avait en outre étudié :

- Des ouvrages sur le mysticisme (son intérêt pour ce phénomène date de $1905^{93}$ et s'intensifie à partir de $1911^{94}$ ): parmi les textes les plus importants qu'il a lus, à part les écrits de certains grands mystiques (notamment les Torrents de Madame Guyon) ${ }^{95}$, sont les Études d'histoire et de psychologie du mysticisme d'Henri Delacroix $(1908)^{96}$ et l'ouvrage de William James, Varieties of Religious Experience $(1902)^{97}$ qui l'ont

88. Cf. L. Lévy-Bruhl, La morale et la science des mours, 1903 et F. Rauh, L'expérience morale, Paris, Alcan, 1903 (cf. Bergson, Correspondances, op. cit., p. 1387 et p. 256 respectivement). Sur le rôle très important de ces deux ouvrages dans le débat concernant le problème moral au début du $\mathrm{XX}^{\mathrm{e}}$ siècle, $\mathrm{cf}$. F. Keck, "Le débat sur La morale et la science des mours de Lucien Lévy-Bruhl (1903). Le problème moral, entre philosophie et sociologie ", in F. Worms (éd.), Le moment 1900 en philosophie, Villeneuve d'Ascq, Presses Universitaires du Septentrion, 2004, p. 373-388. Cependant, Bergson affirme avoir écrit le chapitre des Deux Sources portant sur la morale, sans avoir pensé un seul instant à Lévy-Bruhl, dont il avait lu attentivement le livre mentionné lorsqu'il avait paru (cf. Correspondances, op. cit., p. 1387).

89. Filosofia della pratica, Bari, Laterza, 1909 (cf. Bergson, Correspondances, op. cit., p. 243).

90. La Morale humaine, Paris, Nourry, 1923, $2^{\mathrm{e}}$ éd. revue et corrigée 1928 (cf. Bergson, Correspondances, op. cit., p. 1265 et 1346).

91. Parmi ces ouvrages, il faut notamment mentionner l'Introduction à l'histoire des religions (1929) de Th. Robinson, qui figurait dans la bibliothèque personnelle de Bergson et avait suscité son attention, où nous rencontrons une distinction entre deux types de morale qu'il vaut la peine de comparer avec la distinction bergsonienne entre la morale ouverte et la morale close (cf. Th. Anastassopoulou-Kapoyanni, Causalité et création. Le continu et le discontinu dans l'œuvre d'Henri Bergson, Thèse de doctorat, Université de Paris-Sorbonne, Paris IV, 1990, p. 585-586). - Mentionnons aussi la thèse de M. Pradines (Critique des conditions de l'action. I. L'erreur morale. II. Principes de toute philosophie de l'action, Paris, Alcan, 1909) dont Bergson commente certaines thèses dans ses Correspondances, op. cit., p. 314-315.

92. Cf. Soulez et Worms, Bergson. Biographie, op. cit., p. 135.

93. Cf. J.-L. Vieillard-Baron, «Lettres inédites de Bergson », Annales bergsoniennes, vol. II, op. cit., p. 461 et 463-464.

94. Cf. Soulez et Worms, Bergson. Biographie, op. cit., p. 201 : «depuis 1911 environ, c'est la lecture des mystiques qui le retient et constitue l'objet de son travail philosophique ».

95. Cf. Mélanges, p. 881. Sur le rôle de Mme Guyon dans la conception bergsonienne du mysticisme, cf. Marie Cariou, Lectures bergsoniennes, Paris, P.U.F., «Questions », 1990, p. 107-147 : «Troisième lecture : Bergson entre Mme Guyon et Rousseau ». Cf. aussi, bien entendu, le grand ouvrage de Marie Cariou, Bergson et le fait mystique (AubierMontaigne, 1976).

96. Bergson a présenté cet ouvrage en 1909 devant l'Académie des Sciences Morales et Politiques. Cf. Mélanges, op. cit., p. 788-790. Il en parle également de façon élogieuse dans Les Deux Sources, p. 241, note 1.

97. Cf. les commentaires de Bergson sur cet ouvrage dans Correspondances, op. cit., p. 118 et 128-129 et Mélanges, op. cit., p. 1472. 
largement inspiré ${ }^{98}$. Il a également lu l'ouvrage de Jean Baruzi sur Jean de la Croix ${ }^{99}$. Il cite aussi dans Les Deux Sources d'autres ouvrages sur le mysticisme $^{100}$. Remarquons, toutefois, que Bergson s'est très peu occupé des mysticismes orientaux ${ }^{101}$. On pourrait se demander quel a pu être l'apport de ces lectures sur le mysticisme à la pensée morale de Bergson. Toutefois, cette question ne se pose pas vraiment chez lui, puisque, comme on va le voir, une des deux espèces de morale qu'il distingue, la «morale ouverte », est indissociablement liée à l'expérience mystique ${ }^{\mathrm{102}}$.

- Des études portant sur la psychologie religieuse, tel un rapport de H. Höffding ${ }^{103}$.

- Des livres de sociologie, surtout de sociologie de la religion (en particulier Durkheim, Les formes élémentaires de la vie religieuse ${ }^{104}$ et d'anthropologie sociale (Lucien Lévy-Bruhl ${ }^{105}$, Marcel Mauss etc.)

- Des ouvrages d'histoire des religions, notamment les ouvrages de son collègue Alfred Loisy ${ }^{106}$ et l'étude de Théodore Robinson, Introduction à l'histoire des religions ${ }^{107}$.

98. Cf. Soulez et Worms, Bergson. Biographie, op. cit., p. 373, note 64.

99. Cf. ibid., p. 373, note 65 .

100. Cf. Deux Sources, p. 241, note 1 sur deux ouvrages d'Evelyn Underhill sur le mysticisme. Cf. également ibid., p. 242, note 1 où il se réfère à l'ouvrage dans lequel Pierre Janet étudiait la folie mystique, De l'angoisse à l'extase (Paris, 1928); et p. 262, note 1 où Bergson renvoie à l'ouvrage de M. de Montmorand, Psychologie des mystiques catholiques orthodoxes (Paris, 1920).

101. En avril 1932, après la publication des Deux Sources, il remercie A. Chevrillon en ces termes: «non moins suggestives sont vos observations sur les mysticismes orientaux» (Correspondances, op. cit., p. 1371).

102. Cf. F. Worms, Bergson ou les deux sens de la vie, op. cit., p. 305-306.

103. Cf. Correspondances, op. cit., p. 292-293. Pour d'autres essais connus de Bergson dans ce domaine, cf. aussi ibid., p. 402.

104. Selon L. Kolakowski (Bergson, South Bend, Indiana, St. Augustine's Press, 2001, p. 73), ce livre de Durkheim était l'ouvrage concernant la sociologie de la religion qui était le plus familier à Bergson. Sur l'attitude de Bergson envers Durkheim, en ce qui concerne la religion, je me permets de renvoyer à mon article «Bergson est-il durkheimien dans Les deux sources de la morale et de la religion? », Philosophia (Annales du Centre de Recherche sur la Philosophie Grecque de l'Académie d'Athènes), 36, 2006, p. 230-255.

105. Nous avons vu que Bergson connaissait bien l'ouvrage de Lévy-Bruhl, La morale et la science des mours (cf. supra, note 89). Nous savons par ailleurs qu'il connaissait aussi bien les ouvrages de Lévy-Bruhl, Les fonctions mentales dans les sociétés inférieures, Paris, Alcan, 1910 (cf. Correspondances, op. cit., p. 313-314) et La mentalité primitive, Paris, Alcan, 1922 (cf. Deux Sources, p. 149-158, où Bergson critique les thèses de Lévy-Bruhl sur la mentalité primitive). Sur ce dernier point, cf. l'article de Jean Laporte, « À travers l'actualité philosophique: La mentalité primitive d'après M. Lévy-Bruhl et d'après M. Bergson », La Revue de France, 15 avril 1932.

106. Cf. par exemple Correspondances, p. 252-253, 756-757, 858-859, 872-873, 909-910, 977-978, 1151-1152, etc.

107. Payot, 1929. Cf. supra, note 91. 
e/ Malgré la critique exercée par Bergson dans Les Deux Sources à certaines thèses de Lévy-Bruhl et de Durkheim, Bergson ne considère nullement cet ouvrage comme un livre de polémique, puisqu'il a «toujours été ennemi de la "réfutation" en matière de philosophie ». Bien au contraire, il a «toujours jugé que la philosophie se rapetissait en polémiquant ${ }^{108}$.

\section{Quel degré de certitude Bergson attribue-t-il à ses conclusions dans Les Deux Sources?}

Il affirme constamment se sentir certain de tout ce qu'il avance dans cet ouvrage $^{109}$, de même d'ailleurs que dans ses autres ouvrages ${ }^{110}$, tout en exprimant son mécontentement à propos de la forme achevée des Deux Sources, jugeant que son exposition aurait pu être plus persuasive ${ }^{111}$, et tout en constatant la grande distance entre ce qu'il a fait dans sa carrière et ce qu'il avait voulu faire ${ }^{112}$. Remarquons que, en 1912, Bergson avait déjà déclaré qu'il publierait ses pensées sur la morale uniquement s'il arrivait «à des résultats qui [lui] paraissent aussi démontrables ou aussi "montrables" que ceux de [ses] autres travaux ${ }^{113}$. Et nous connaissons la valeur qu'avait un tel engagement de la part de Bergson.

Par conséquent, nous comprenons pourquoi il réagit vigoureusement lorsqu'on présente Les Deux Sources « comme une série de remarques sur la morale et sur la religion» qu'on pourrait combattre avec d'autres remarques ${ }^{114}$. Bergson considère Les Deux Sources comme un livre de philosophie, et non comme un exposé de ses choix ou de ses opinions

108. Correspondances, op. cit., p. 1387-1388.

109. Cf. ibid., p. 1388-1389 : «Vous avez d'ailleurs bien raison de donner à entendre qu'il y a dans ce livre [dans Les Deux Sources] des lacunes. Elles tiennent, le plus souvent, à ce que je me tais quand je ne vois plus clair, ne voulant pas mêler à des conclusions dont je me sens certain des opinions simplement personnelles et conjecturales » (c'est nous qui soulignons). Cf. supra, note 83. Cf. également ibid., p. 1377 : «Une seule chose me paraît certaine, c'est que Dieu doit être dit transcendant au monde, puisque Dieu, tel que le mystique l'aperçoit, pourrait se passer du monde, tandis que le monde, si le mysticisme a raison, ne peut se passer de Dieu ».

110. Cf. ibid., p. 1184 : « [...] la certitude toute particulière que j'attribue, à tort ou à raison, aux conclusions que $\mathrm{j}$ 'apporte $»$.

111. Cf. ibid., p. 1363 : «À me relire imprimé, j’ai éprouvé plus de mécontentement encore que devant mon manuscrit. Justement parce que je me sens sûr de ce que j'avance, je m'en veux de ne pas l'avoir exposé d'une manière persuasive [...] Cette absurde maladie m'aura empêché de dire des choses comme je voulais les dire ». Signalons cependant que Bergson s'était exprimé à peu près de la même manière à propos de Matière et mémoire ; cf. ibid., p. 37.

112. Cf. ibid., p. 1362 : «Hélas ! arrivé au terme de ma carrière, je mesure exactement la distance entre ce que j'ai fait et ce que j'avais voulu faire. Elle est grande [...]»Cf. aussi ibid., p. 1280, 1281 et 1284 .

113. Cf. Mélanges, op. cit., p. 964.

114. Cf. Camille de Belloy, «Une mise au point de Bergson sur Les Deux Sources », Annales bergsoniennes, vol. I, op. cit., p. 135. 
personnels. En général, il faisait tout ce qui était en son pouvoir pour qu'on ne confonde pas «le Bergson philosophe » avec «le Bergson qui exprime un certain sentiment personnel ${ }^{115}$.

Il faut pourtant souligner que Bergson distingue le genre de certitude qu'on peut obtenir en métaphysique de celui qu'on demande aux mathématiques et nous incite à nous «débarrasser de ce "mathématisme universel" dont [notre philosophie] reste pénétrée ${ }^{116}$.

Mon but par la suite - suivant les instructions de Bergson lui-même sera de «démêler les idées essentielles [de la morale des Deux Sources] et d'en marquer l'enchaînement ${ }^{117}$.

\section{Quelles sont les deux sources de la morale selon Bergson ?}

\section{A/ Pression sociale - obligation (morale close, âme close) $)^{118}$}

Selon Bergson, «le problème moral par excellence est d'expliquer l'obligation. Aucun philosophe, à mon avis, n'y a réussi. C'est pourquoi je dois traiter de l'obligation morale à part et d'abord ${ }^{119}$. Son point de départ est la constatation que l'obligation est inéluctablement présente dans toute société, dans toute vie humaine. Nous trouvons l'obligation au fond de notre conscience $^{120}$. «Il est nécessaire qu'il y ait des obligations ${ }^{121}$. «L'obligation envisagée comme [...] simple forme, sans matière [...] est ce qu'il y a d'irréductible, et de toujours présent encore, dans notre nature morale $»^{122}$.

En quoi consiste l'obligation? Quelle est sa fonction ? En recourant à l'étymologie du mot, Bergson affirme que «l'obligation [...] nous lie aux autres membres de la société ${ }^{123}$. Sa fonction est la sauvegarde de la cohésion sociale ${ }^{124}$.

115. Cf. par exemple Correspondances, op. cit., p. 1185.

116. Ibid., p. 442.

117. Ibid., p. 1388.

118. Bergson avait déjà thématisé la différence entre l'ouvert et le fermé, notions entre lesquelles il y a une différence de nature et non pas simplement une différence de degré, dès L'Evolution créatrice (cf. Euvres, p. 718).

119. Cf. dans les Annales bergsoniennes, vol. I, op. cit., p. 133 le texte de deux feuillets glissés par Bergson dans son exemplaire de l'ouvrage de Loisy, Y a-t-il deux sources de la morale et de la religion?

120. Cf. Deux Sources, p. 84.

121. Ibid., p. 24.

122. Ibid., p. 84.

123. Ibid.

124. Cf. ibid., p. 18 : «L'obéissance de tous à des règles, même absurdes, assure à la société une cohésion plus grande » et ibid., p. $84:$ « [...] par le besoin de conservation sociale». 
Par quel genre de lien l'obligation nous lie-t-elle aux autres membres de la société ? «L'obligation [...] qui [...] nous lie aux autres membres de la société, est un lien du même genre que celui qui unit les unes aux autres les fourmis d'une fourmilière ou les cellules d'un organisme ${ }^{125}$, avec la différence fondamentale que l'homme est un être intelligent, comme nous le verrons un peu plus loin.

Certains parmi les exemples d'obligation présentés par Bergson sont les suivants : (a) L'obéissance aux interdictions de nos parents et de nos maîtres lorsque nous étions enfants ${ }^{126}$. (b) «La consigne militaire, qui est un ordre non motivé et sans réplique, dit bien qu' "il faut parce qu'il faut" " ${ }^{127}$.

Soulignons que Bergson commence Les Deux Sources, sans introduction, par la phrase suivante :

Le souvenir du fruit défendu est ce qu'il y a de plus ancien dans la mémoire de chacun de nous, comme dans celle de l'humanité. Nous nous en apercevrions si ce souvenir n'était recouvert par d'autres, auxquels nous préférons nous reporter ${ }^{128}$.

En quoi consiste la différence entre l'obligation et la nécessité ? Bergson précise que l'obligation implique l'intelligence :

L'obligation [...] qui [...] nous lie aux autres membres de la société, est un lien du même genre que celui qui unit les unes aux autres les fourmis d'une fourmilière ou les cellules d'un organisme. C'est la forme que prendrait ce lien aux yeux d'une fourmi devenue intelligente comme un homme, ou d'une cellule organique devenue aussi indépendante dans ses mouvements qu'une fourmi intelligente ${ }^{129}$.

Dans un autre passage des Deux Sources, il est clair que l'obligation implique la liberté : à l'inverse de l'instinct social de la fourmi ou de la « façon dont la cellule d'un corps vivant fonctionne pour le plus grand bien de l'ensemble » - «dans un cas comme dans l'autre, il n'y a proprement obligation; il y aurait plutôt nécessité »-, toute obligation implique la liberté ${ }^{130}$.

En effet, lorsque Bergson propose une définition de l'obligation, il est évident qu'elle implique intelligence, choix, liberté :

125. Ibid., p. 84.

126. Cf. ibid., p. 1.

127. Ibid., p. 19.

128. Ibid., p. 1.

129. Ibid., p. 84.

130. Cf. ibid., p. 24. 
L'obligation nous apparaît comme la forme même que la nécessité prend dans le domaine de la vie quand elle exige, pour réaliser certaines fins, l'intelligence, le choix, et par conséquent la liberté ${ }^{131}$.

Une des distinctions essentielles proposées par Bergson dans Les Deux Sources à propos de l'obligation est celle entre les obligations particulières et «le tout de l'obligation». Il soutient que, à la surface, au sommet, nous sommes conscients des obligations particulières. Mais, si nous creusons au fond, si nous descendons, nous arrivons à «l'obligation en général » ou au «tout de l'obligation qui est à la base ${ }^{132}$. Comment définit-il ce «tout de l'obligation »?

Représentez-vous l'obligation comme pesant sur la volonté à la manière d'une habitude, chaque obligation traînant derrière elle la masse accumulée des autres et utilisant ainsi, pour la pression qu'elle exerce, le poids de l'ensemble : vous avez le tout de l'obligation pour une conscience morale simple, élémentaire. C'est l'essentiel ; et c'est à quoi l'obligation pourrait à la rigueur se réduire, là même où elle atteint sa complexité la plus haute ${ }^{133}$.

Une des conséquences majeures de la théorie bergsonienne du « tout de l'obligation » est l'interpénétration des obligations :

En raison de la solidarité de nos obligations entre elles, et parce que le tout de l'obligation est immanent à chacune de ses parties, tous les devoirs se colorent de la teinte qu'a prise exceptionnellement tel ou tel d'entre eux ${ }^{134}$.

Suivant une autre thèse majeure des Deux Sources l'obligation fonctionne dans le cas de l'homme de manière analogue à la façon dont fonctionne l'instinct dans le cas des animaux : l'ensemble des habitudes, « je veux dire l'habitude de contracter ces habitudes, étant à la base même des sociétés et conditionnant leur existence, aura une force comparable à celle de l'instinct, et comme intensité et comme régularité. C'est là précisément ce que nous avons appelé "le tout de l'obligation" ${ }^{135}$. Un peu plus loin, Bergson qualifiera le "tout de l'obligation" $d$ '"instinct virtuel" : "Aucune obligation n'étant de nature instinctive, le tout de l'obligation eût été de l'instinct, si les sociétés humaines n'étaient en quelque sorte lestées de variabilité et d'intelligence. C'est un instinct virtuel ${ }^{136}$. Remarquons ici

\section{Ibid.}

132. Ibid.

133. Ibid., p. 19

134. Ibid., p. 13. Rappelons que, à l'opposé de la juxtaposition qui caractérise la liaison des objets dans l'espace, Bergson considère l'interpénétration comme une des caractéristiques essentielles de la durée psychologique dans l'Essai sur les données immédiates de la conscience (Euvres, p. 84-85, 88-89 et 107-109).

135. Deux Sources, p. 21.

136. Ibid., p. 22-23. 
que, à propos de la religion, Bergson qualifiera d'«instinct virtuel » la fonction fabulatrice ${ }^{137}$, et il en est à peu près de même du «double quasiinstinct de commandement et d'obéissance qui définit la société naturelle comme intrinsèquement hiérarchique et inégalitaire » dans le quatrième chapitre des Deux Sources. ${ }^{138}$

Quel rôle jouent le naturel et l'acquis dans l'explication du "tout de l'obligation" ? Étant donné que Bergson rejette la théorie de l'hérédité de l'acquis - ce rejet constitue même « un des deux ou trois points essentiels des Deux Sources ${ }^{139}$, mais aussi de L'Évolution créatrice ${ }^{140}$ - il soutient que, pour expliquer "le tout de l'obligation", il faut nous reporter au naturel et non à l'acquis, car le naturel n'est pas «écrasé par les habitudes acquises qui se sont accumulées sur lui pendant des siècles de civilisation, mais il se maintient en fort bon état, très vivant, dans la société la plus civilisée ${ }^{141}$.

Quelles sont les sources de l'obligation? Selon Bergson l'obligation ne peut pas découler de la raison, de la spéculation. On ne peut pas déduire une morale d'une doctrine; il s'agit même d'une thèse essentielle à ses yeux ${ }^{142}$. "Aucune spéculation ne créera une obligation ou rien qui y ressemble ; peu m'importe la beauté de la théorie, je pourrai toujours dire que je ne l'accepte pas; et même si je l'accepte, je prétendrai rester libre de me conduire à ma guise ${ }^{143}$.

L'obligation provient de la société ; mais, compte tenu de la distinction fondamentale opérée par Bergson dans Les Deux Sources entre la société close et la société ouverte, nous devons préciser que l'obligation provient de la société close. $\mathrm{Au}$ fond de l'obligation morale se trouve l'exigence sociale $^{144}$. «Une bonne moitié de notre morale comprend des devoirs dont le caractère obligatoire s'explique en dernière analyse par la pression de la société sur l'individu ${ }^{145}$. Pourtant, dans d'autres endroits des Deux Sources Bergson soutient que l'obligation se rattache aux phénomènes les plus généraux de la vie : étant donné que, selon Bergson, premièrement, «le tout de l'obligation» est un «instinct virtuel», deuxièmement, il faut nous reporter sans cesse «à ce qu'eût été l'obligation si la société humaine avait

137. Cf. ibid., p. 114, 124 et 126.

138. Cf. F. Worms, Bergson ou les deux sens de la vie, op. cit., p. 295-296. Sur le sens et le rôle de la notion d' «instinct virtuel » dans Les Deux Sources, cf. les analyses de F. Worms, ibid., p. 278-281.

139. Cf. dans les Annales bergsoniennes, vol. I, op. cit., p. 134 le texte de deux feuillets glissés par Bergson dans son exemplaire de l'ouvrage de Loisy, Y a-t-il deux sources de la morale et de la religion?

140. L'Évolution créatrice, p. 79-85 (p. 561-567 des Euvres).

141. Deux Sources, p. 25.

142. Cf. ibid., p. 18 : «l'essence de l'obligation est autre chose qu'une exigence de la raison. C'est tout ce que nous avons voulu suggérer jusqu'à présent ».

143. Ibid., p. 45.

144. Cf. ibid., p. 25.

145. Ibid., p. 46 (c'est nous qui soulignons). 
été instinctive au lieu d'être intelligente ${ }^{146}{ }^{46}$, et troisièmement, nous devons penser à cette société instinctive "comme à un pendant de la société intelligente, si l'on ne veut pas s'engager sans fil conducteur dans la recherche des fondements de la morale ${ }^{147}$, la conclusion s'impose : «De ce point de vue, l'obligation perd son caractère spécifique. Elle se rattache aux phénomènes les plus généraux de la vie ${ }^{148}$.

Entre les deux sources - du moins en apparence - de l'obligation, la société et la vie, laquelle est la source primordiale ? Cela ne pose pas de problème suivant Bergson ; c'est la vie, car «le social est au fond du vital ». Tout se réduit à la biologie, «au sens très compréhensif du terme ${ }^{149}$. Le texte le plus explicite à ce propos est le suivant :

Au sujet de cette obligation, j'ai essayé d'établir que les philosophes n'arrivent jamais à l'expliquer, à l'engendrer, parce que, sans s'en apercevoir, ils se la donnent d'abord. Elle est là, et dès lors ils peuvent déduire la morale de n'importe quel mobile, pris au hasard. Mais aucune de leurs déductions n'explique l'obligation. Je dis moi, qu'elle est là, en effet. Mais je montre pourquoi, comment, etc. Elle est là, parce que c'est une donnée biologique. La correspondance entre l'instinct social des hyménoptères et les sociétés humaines est une de mes constatations fondamentales. [...] La première base de l'obligation est donc biologique, et non pas sociologique (au sens de Durkheim). C'est une grosse erreur que de voir du Durkheim dans mon premier chapitre [des Deux Sources] ${ }^{150}$.

Comment Bergson réagit-il aux reproches de réductionnisme et plus particulièrement de biologisme faits à cette théorie ? Il ne s'en inquiète pas, car l'obligation, dont la première base est biologique et non sociologique, «n'est que la donnée première. Il y a bien autre chose [...] l'aspiration, qui prolonge l'obligation primitive ${ }^{151}$. On peut toutefois rétorquer que le problème subsiste, car, lorsque Bergson développe sa pensée, il soutient que «"pression sociale" et "élan d'amour" ne sont que deux manifestations complémentaires de la vie ${ }^{152}$.

146. Ibid., p. 23.

147. Ibid.

148. Ibid.

149. Cf. ibid., p. 103. Soulignons que ce n'est pas une pratique courante chez Bergson d' « étendre beaucoup le sens des mots ». C'est la raison principale de son désaccord avec la thèse soutenue par John Dewey dans son article «Perception and organic action » : «Il me serait impossible de définir toute la réalité en terme d'action, à moins d'étendre beaucoup le sens de ce mot » (Correspondances, op. cit., p. 498 ; c'est Bergson qui souligne).

150. Cf. dans les Annales bergsoniennes, vol. I, op. cit., p. 133 le texte de deux feuillets glissés par Bergson dans son exemplaire de l'ouvrage de Loisy, Y a-t-il deux sources de la morale et de la religion? (c'est Bergson qui souligne).

151. Ibid., p. 134.

152. Deux Sources, p. 98. 
Quelle représentation de la société est immanente à la pression considérée en elle-même ? La représentation d'une société close dont le seul but est de se conserver ${ }^{153}$.

Quant au sentiment qui caractérise la conscience de cet ensemble d'obligations pures, à supposer que toutes sont remplies, c'est «un état de bien-être individuel et social comparable à celui qui accompagne le fonctionnement normal de la vie. Il ressemblerait au plaisir plutôt qu'à la joie $\gg{ }^{154}$.

\section{B/ Aspiration ou appel (morale ouverte, âme ouverte)}

À part l'obligation qui est inéluctablement présente dans la vie des hommes et dont la source primordiale est biologique, Bergson soutient qu'il y a une autre donnée également empirique : «il y a bien autre chose ... l'aspiration ${ }^{155}$ de la conscience individuelle ${ }^{156}$. Il constate empiriquement l'existence de la morale ouverte : «De tout temps ont surgi des hommes exceptionnels en lesquels cette morale - la morale complète, «qu'on ferait mieux d'appeler absolue $»-s^{\prime}$ incarnait ${ }^{157}$.

Quel est le rapport entre l'aspiration et l'obligation (pression sociale) ? Selon Bergson l'aspiration "prolonge l'obligation primitive et lui emprunte ce qu'elle a d'impératif ${ }^{158}$.

Qui a incarné la morale complète ou absolue? «Avant les saints du christianisme, l'humanité avait connu les sages de la Grèce, les prophètes d'Israël, les Arahants du bouddhisme et d'autres encore ${ }^{159}$. Nous remarquons qu'il y a des représentants de toutes les grandes civilisations. «Le héros archétype de cette morale ouverte est Socrate [...] qui communique son élan à ses disciples à un niveau "supra-intellectuel", qui est "aspiration, intuition et émotion" " ${ }^{160}$. Ailleurs Bergson parle de «fondateurs et réformateurs de religions, mystiques et saints, héros obscurs de la vie morale que nous avons pu rencontrer sur notre chemin et qui égalent à nos

153. Cf. ibid., p. 49 : «Immanente à la première [la pression] est la représentation d'une société qui ne vise qu'à se conserver: le mouvement circulaire où elle entraîne avec elle les individus, se produisant sur place, imite de loin, par l'intermédiaire de l'habitude, l'immobilité de l'instinct ».

154. Ibid.

155. Cf. dans les Annales bergsoniennes, vol. I, op. cit., p. 134 le texte des feuillets glissés par Bergson dans son exemplaire de l'ouvrage cité de Loisy, $Y$ a-t-il deux sources de la morale et de la religion?

156. Cf. Deux Sources, p. $84:$ : [...] par une aspiration de la conscience individuelle».

157. Ibid., p. 29.

158. Cf. dans les Annales bergsoniennes, vol. I, op. cit., p. 134 le texte des feuillets glissés par Bergson dans son exemplaire de l'ouvrage de Loisy, $Y$ a-t-il deux sources de la morale et de la religion?

159. Deux Sources, p. 29.

160. J.-L. Vieillard-Baron, Bergson, Paris, P.U.F., «Que sais-je ?», $2^{\mathrm{e}}$ édition 1993, p. 87. 
yeux les plus grands ${ }^{161}$. Si on voulait à tout prix citer quelques exemples de ces derniers, signalons que, en 1939, un peu avant la mort de Bergson, dans un texte dédié à Charles Péguy, son disciple et ami, nous constatons que ce dernier «a rejoint, aux yeux de Bergson, les héros et les saints dont il est question dans Les Deux Sources ${ }^{162}$. Nous pensons également à la façon dont le métaphysicien Jean Wahl et surtout le talmudiste Mordechaï Chouchani ont inspiré, chacun à sa manière, la vie et la pensée de Lévinas ${ }^{163}$.

Le problème le plus important concernant la morale ouverte est le suivant : Comment expliquer l'écho que suscitent les grands mystiques, les héros et les saints, comment expliquer l'influence qu'ils exercent sur tous les autres hommes ? ${ }^{164}$ "Comment les mystiques ont-ils exercé une telle influence sur l'orientation morale de l'humanité ${ }^{165}$ ? Selon Bergson, la force que les hommes de bien exceptionnels possèdent sur les autres hommes « est ... psychologique, mais non pas rationnelle, elle ne repose plus cependant sur l'instinct, mais sur l'émotion ${ }^{166}$. En effet, la spécificité essentielle de la morale ouverte est qu'elle traduit un certain état émotionnel et, par conséquent, on ne cède plus ici à une pression, mais à un attrait ${ }^{167}$. Les héros en morale, les saints, les grands mystiques communiquent de leur ardeur et entraînent derrière eux, à travers leur exemple, les hommes ${ }^{168}$.

Les initiateurs en morale procèdent comme la musique qui exprime des émotions :

Que la musique exprime la joie, la tristesse, la pitié, la sympathie, nous sommes à chaque instant ce qu'elle exprime. Non seulement nous, mais beaucoup d'autres, mais tous les autres aussi. Quand la musique pleure, c'est l'humanité, c'est la nature entière qui pleure avec elle. À vrai dire, elle n'introduit pas ces sentiments en nous ; elle nous introduit plutôt en eux, comme des passants qu'on pousserait dans une danse. Ainsi procèdent les initiateurs en morale. La vie a pour eux des résonances de sentiment insoupçonnées, comme en pourrait donner une symphonie nouvelle; ils nous font entrer avec eux dans cette musique, pour que nous la traduisions en mouvement ${ }^{169}$.

161. Deux Sources, p. 47.

162. Soulez et Worms, Bergson. Biographie, p. 254-255 : il l'appelle «génie mystique ».

163. Cf. Salomon Malka, Lévinas. La vie et la trace, Paris, Albin Michel, «Spiritualités vivantes », 2005, p. 157-169.

164. Cf. F. Worms, Bergson ou les deux sens de la vie, op. cit., p. 302.

165. Bergson, Correspondances, p. 1236.

166. F. Worms, Bergson ou les deux sens de la vie, p. 302-303. L'auteur résume ici l'argument développé par Bergson dans Les Deux Sources, p. 35-49 (p. 1007-1118 des Cuvres).

167. Cf. Deux Sources, p. 46.

168. Cf. ibid., p. 98 : «nous sentons qu'ils nous communiquent de leur ardeur et qu'ils nous entraînent dans leur mouvement ».

169. Ibid., p. 36. 
N'y a-t-il pas un obstacle pratique dans cette théorie de Bergson? Qu'est-ce qui se passe si on n'a pas rencontré ces grands hommes de bien ? Cette objection ne pose aucun problème pour Bergson, car ce n'est pas seulement $[a]$ la parole d'un grand mystique qui trouve un écho chez tel ou tel entre nous, mais également [b] la parole «de quelqu'un de ses imitateurs $\gg{ }^{170}$. Enfin, si la parole du grand mystique ou d'un de ses imitateurs trouve cet écho chez nous, «n'est-ce pas que [c] il peut y avoir en nous un mystique qui sommeille et qui attend seulement une occasion de se réveiller ? ${ }^{171}$ Par conséquent, dans le cas de la morale ouverte, de la morale de l'aspiration, la personne « répond à l'appel d'une personnalité, qui peut être celle d'un révélateur de la vie morale, ou celle d'un de ses imitateurs, ou même, dans certaines circonstances, la sienne ${ }^{172}$.

Par ailleurs, nous pouvons ressusciter par la pensée ces grands hommes de bien ${ }^{173}$. Ailleurs Bergson souligne que l'exemple des grands initiateurs en morale « a fini par entraîner les autres, au moins en imagination ${ }^{174}$.

Tous les hommes sont-ils entraînés par l'exemple de ces personnalités exceptionnelles ? Tous ressentent-ils leur appel ? Bergson est obligé de constater, à propos des grands mystiques, que cela n'est pas toujours le $\operatorname{cas}^{175}$, sans pourtant que sa théorie en pâtisse : Comme il y a des gens qui ne sont pas émus par la musique, pour qui la musique est du bruit, ainsi il y a des gens qui sont sourds à l'appel des grands mystiques.

Mais pourquoi les grands hommes de bien agissent-ils, pourquoi ne se contentent-ils pas de la simple contemplation? Les grands mystiques, «sûrs d'eux-mêmes, parce qu'ils sentent en eux quelque chose de meilleur qu'eux, ils se révèlent grands hommes d'action, à la surprise de ceux pour qui le mysticisme n'est que vision, transport, extase. Ce qu'ils ont laissé couler à l'intérieur d'eux-mêmes, c'est un flux descendant qui voudrait, à travers eux, gagner les autres hommes : le besoin de répandre autour d'eux ce qu'ils ont reçu, ils le ressentent comme un élan d'amour ${ }^{176}$.

Une des thèses essentielles de Bergson sur la morale ouverte est que « la morale de l'Évangile est essentiellement celle de l'âme ouverte ${ }^{17 T}$; il se réfère surtout ici au Sermon sur la montagne ${ }^{178}$, en nous faisant penser aux

170. Ibid., p. 102.

171. Ibid.

172. Ibid.

173. Cf. ibid., p. 98.

174. Ibid., p. 56.

175. Cf. ibid., p. 102 : «Si la parole d'un grand mystique, ou de quelqu'un de ses imitateurs, trouve un écho chez tel ou tel d'entre nous [...]» (c'est nous qui soulignons).

176. Ibid., p. 101-102.

177. Ibid., p. 57.

178. Cf. ibid., p. 57-58. Sur le rôle fondamental du «Christ des Évangiles» dans la conception bergsonienne de la morale ouverte, cf. F. Worms, Bergson ou les deux sens de la vie, op. cit., p. 316-317. 
pages célèbres de Rousseau sur la supériorité de Jésus par rapport à Socrate dans la «Profession de foi du vicaire savoyard», au cœur de son chef d'œuvre pédagogique, Émile.

Pour mieux comprendre cette identification de la morale de l'âme ouverte avec la morale de l'Évangile, sur laquelle Bergson revient souvent ${ }^{179}$, il faut la confronter avec sa thèse, formulée bien entendu après la publication des Deux Sources, suivant laquelle le type de la morale close est la morale allemande hitlérienne ${ }^{180}$.

Quel rapport entretiennent les deux espèces de morale avec la nature ? Selon Bergson, tandis que la «morale close » est naturelle, la morale ouverte rompt avec la nature ${ }^{181}$. Mais en réalité, elle ne rompt pas avec le tout de la Nature, mais seulement avec une certaine nature (et ici Bergson a recours à la fameuse distinction entre Nature naturante et Nature naturée que nous rencontrons notamment dans l'Éthique de Spinoza ${ }^{182}$ ): «En allant de la solidarité sociale à la fraternité humaine, nous rompons avec une certaine nature, mais non pas avec toute nature. On pourrait dire, en détournant de leur sens les expressions spinozistes, que c'est pour revenir à la Nature naturante que nous nous détachons de la Nature naturée ${ }^{183}$.

Un des points communs entre la morale close et la morale ouverte est que, comme nous l'avons vu à propos des obligations ${ }^{184}$ - mais non à propos des maximes de la morale close - les maximes de la morale ouverte s'interpénètrent : "les maximes de cette seconde morale n'opèrent pas isolément, comme celles de la première : dès que l'une d'elles, cessant d'être abstraite, se remplit de signification et acquiert la force d'agir, les autres tendent à en faire autant ; finalement toutes se rejoignent dans la chaude émotion qui les laissa jadis derrière elle et dans les hommes, redevenus vivants, qui l'éprouvèrent ${ }^{185}$.

Bergson mentionne au moins trois autres différences entre la morale ouverte et la morale close. Premièrement, à l'opposé de la morale de la

179. Cf. Correspondances, p. 1380: «j'ai exposé dans mon premier chapitre [des Deux Sources] que la morale de l'Évangile était celle de l'âme ouverte, de la société ouverte, - ce qui implique que tout progrès de l'humanité dans le sens de l'“ouverture" se trouvera être du christianisme par définition même, si je puis m'exprimer ainsi ».

180. Cf. J. Chevalier, Entretiens avec Bergson, p. 228 : «Quant à l'hitlérisme, il est une démonstration éclatante de la thèse que j'avais soutenue dans Les Deux Sources : à savoir que la morale close (dont le type est la morale allemande hitlérienne) est étroitement liée à une conception païenne du monde et des choses et à une résurrection du culte de Wotan. Le christianisme seul nous a appris à regarder par-dessus les frontières et à étendre notre amour à tous les hommes» (citation puisée dans Soulez et Worms, Bergson. Biographie, op. cit., p. 383, note 64).

181. Cf. Deux Sources, p. 54-56.

182. Cf. Éthique I, XXIX, scolie.

183. Deux Sources, p. 56.

184. Cf. supra, note 135.

185. Cf. Deux Sources, p. 47. 
pression, à laquelle est immanente «la représentation d'une société qui ne vise qu'à se conserver », la morale de l'aspiration contient implicitement le sentiment d'un progrès ${ }^{186}$. Deuxièmement, «la première morale est relativement facile à formuler, mais non pas la seconde. Notre intelligence et notre langage portent en effet sur des choses ; ils sont moins à leur aise pour représenter des transitions ou des progrès ${ }^{187}$. Troisièmement, à l'opposé de l'âme close, qui peut aimer sa famille et sa patrie mais non l'humanité entière, l'âme ouverte aime l'humanité, mais elle aime aussi les animaux, les plantes, toute la nature ${ }^{188}$.

\section{Les deux espèces de morale (close et ouverte) existent-elles isolément dans la réalité ou sont-elles mêlées ?}

Il est très important de retenir que, selon Bergson, la morale close et la morale ouverte n'ont jamais existé et n'existeront jamais à l'état pur: il s'agit d'idées limites. Leur statut est celui de «limites extrêmes ${ }^{189}$. Le passage à la limite en bas est l'obligation pure, tandis qu'en haut est l'aspiration ${ }^{190}$. Les deux limites extrêmes « ont plutôt un intérêt théorique; il n'arrive guère qu'elles soient réellement atteintes ${ }^{191}$. «Les deux morales [...] ne se présentent plus à l'état pur. La première a passé à l'autre quelque chose de sa force de contrainte; la seconde a répandu sur la première quelque chose de son parfum. Nous sommes en présence d'une série de gradations et de dégradations, selon qu'on parcourt les prescriptions de la morale en commençant par une extrémité ou par l'autre ${ }^{192}$.

186. Ibid., p. 49.

187. Ibid., p. 57.

188. Cf. ibid., p. 50 : «Qu'une âme ainsi mobilisée soit plus encline à sympathiser avec les autres âmes, et même avec la nature entière $[\ldots] »$.

189. Cf. dans les Annales bergsoniennes, vol. I, op. cit., p. 134 le texte des feuillets glissés par Bergson dans son exemplaire de l'ouvrage de Loisy, $Y$ a-t-il deux sources de la morale et de la religion?

190. Deux Sources, p. 29.

191. Ibid., p. 48. Cependant, dans les feuillets glissés par Bergson dans son exemplaire de l'ouvrage de Loisy, Y a-t-il deux sources de la morale et de la religion? (cf. Annales bergsoniennes, vol. I, op. cit., p. 134), l'auteur des Deux Sources écrit : « Mes "deux morales" sont des limites extrêmes, atteintes isolément dans la pratique par les sociétés primitives et par les grands saints, mais ordinairement mêlées ».

192. Deux Sources, p. 48. Cf. également ibid., p. 47: «Ces deux morales juxtaposées semblent maintenant n'en plus faire qu'une, la première ayant prêté à la seconde un peu de ce qu'elle a d'impératif et ayant d'ailleurs reçu de celle-ci, en échange, une signification moins étroitement sociale, plus largement humaine». 


\section{Morale intermédiaire entre la morale close et la morale ouverte : l'âme qui s'ouvre}

Comme il arrive dans la plupart des distinctions opérées par Bergson, entre la morale close et la morale ouverte il y a possibilité de transition : «Entre l'âme close et l'âme ouverte il y a l'âme qui s'ouvre. Entre l'immobilité de l'homme assis, et le mouvement du même homme qui court, il y a son redressement, l'attitude qu'il prend quand il se lève. Bref, entre le statique et le dynamique, on observe en morale une transition ${ }^{193}$.

En quoi consiste cette transition? Comment faut-il la comprendre ? «Cette transition ne doit pas être prise comme une possibilité de synthèse du clos et de l'ouvert, comme une possibilité de dialectisation de la morale bergsonienne. Elle est [...] une transition qui n'opère aucune réduction et qui garde intacte la distance séparant la morale close de la morale ouverte ${ }^{194}$.

\section{La distinction fondamentale de Bergson en matière de morale}

Selon Frédéric Worms, la distinction fondamentale de Bergson en matière de morale et de religion n'est pas vraiment celle entre la morale close et la morale ouverte, mais plutôt :

la distinction entre le clos et l'ouvert comme distinction transversale dans la morale et la religion, entre toutes les morales et toutes les religions [...] Il ne s'agira pas en effet de distinguer entre une morale et une autre, ou entre une religion et une autre, mais entre le clos et l'ouvert dans toutes: tel est l'acte simple et fondamental de ce livre [...] En effet, le clos et l'ouvert désignent moins une morale et une religion, que deux manières de distinguer entre les morales et les religions [...] La thèse de Bergson sera donc bien que, dans chaque morale et dans chaque religion (peut-être aussi dans chaque philosophie), il y a une part de clôture et une part d'ouverture, qui sont moins des données abstraites que des forces concrètes, qui appellent non seulement des distinctions, mais des actes ${ }^{195}$.

193. Ibid., p. 62.

194. Georges Mourélos, Bergson et les niveaux de réalité, Paris, P.U.F., «Bibliothèque de philosophie contemporaine », 1964, p. 167.

195. F. Worms, Bergson ou les deux sens de la vie, op. cit., p. 266-267. 